\title{
Caracterização isoenzimática de oito acessos de Erva-de-bicho ${ }^{1}$
}

\author{
Reginalda C. Lopes; Vicente Wagner D. Casali; Luiz Cláudio A. Barbosa; Paulo R. Cecon
}

UFV, Depto. Fitotecnia, 36.570-000 - Viçosa-MG; E-mail: vwcasali@mail.ufv.br

\begin{abstract}
RESUMO
Na caracterização de oito acessos de Polygonum punctatum Ell., utilizou-se a técnica de eletroforese horizontal em gel de amido a $12 \%$. Determinaram-se os fenótipos isoenzimáticos da malato desidrogenase $(\mathrm{MDH})$, fosfatase ácida (ACP), peroxidase (PO), glutamato desidrogenase (GDH), leucina aminopeptidase (LAP), esterase (EST), álcool desidrogenase (ADH), glutamato oxaloacetato transaminase (GOT), isocitrato desidrogenase (IDH) e chiquimato desidrogenase (SKDH), utilizando extratos de folhas jovens de cada acesso. A eletroforese foi conduzida em géis de amido de milho e amido hidrolisado. $\mathrm{O}$ amido de milho mostrou resolução satisfatória na separação das bandas isoenzimáticas da IDH, ADH e MDH. No entanto, para os demais sistemas, o amido hidrolisado apresentou resolução superior à do amido de milho. Todos os sistemas enzimáticos estudados, com exceção do sistema LAP, apresentaram elevado polimorfismo, permitindo estabelecer padrões individuais em todos os acessos. Isso demonstrou o potencial das isoenzimas como marcadores genéticos no gênero Polygonum.
\end{abstract}

Palavras-chave: Polygonum punctatum Ell., isoenzimas, diversidade genética.

\begin{abstract}
Isozimatic characterization in eight accesses of dotted Smartweed

The technique of horizontal electrophoresis of $12 \%$ starch gel was used to characterize eight accesses of Polygonum punctatum Ell. The isozymatic phenotypes of the malate dehydrogenase (MDH), acid phosphatase (ACP), peroxidase (PO), glutamate dehydrogenase $(\mathrm{GDH})$, leucine aminopeptidase (LAP), esterase (EST), alcohol dehydrogenase (ADH), glutamate oxalacetate transaminase (GOT), isocitrate dehydrogenase (IDH) and shikimate dehydrogenase (SKDH) were determined using young leaves extracts of each accession. The electrophoresis was performed in corn starch and hydrolysed starch gels. The corn starch showed a satisfactory resolution, for separation of the isozymics bands of IDH, ADH and $\mathrm{MDH}$. However, for the other systems, the hydrolysed starch presented better resolution. All enzyme systems studied, except for LAP, presented high polymorphism, allowing to establish individual patterns in all accesses, demonstrating the potential of the isozymes as genetic markers for the Polygonum.
\end{abstract}

Keywords: Polygonum punctatum Ell., isozymes, genetic diversity.

\section{(Recebido para publicação em 8 de março de 2002 e aceito em 6 de junho de 2003)}

$\mathrm{O}$ estudo dos padrões isoenzimáticos tem sido amplamente utilizado na caracterização de espécies, cultivares e de acessos de bancos de germoplasma. As técnicas isoenzimáticas têm sido também de grande importância como auxiliar na identificação quimiotaxonômica de espécies, principalmente medicinais. No estudo dos padrões isoenzimáticos de duas variedades botânicas de Ocimum nudicaule Benth., Figueiredo-Ribeiro et al. (1986) observaram que as diferenças encontradas confirmaram a existência das duas variedades, anteriormente proposta segundo características químicas e morfológicas.

A introdução da técnica de eletroforese de isoenzimas nos estudos de populações e de evolução também foi de grande importância, porque além de iniciar a era da genética molecular, forneceu um meio direto de avaliação da variação genética (Torggler, 1995).

No Brasil, há um enorme potencial de plantas medicinais usadas popularmente e ainda pouco estudadas pela comunidade científica. Dentro da família Polygonaceae, destaca-se a espécie Polygonum punctatum Ell., conhecida popularmente como erva-de-bicho, acataia, cataia, capiçoba, pimenta-dobrejo ou pimenta-da-água. Essa espécie é perene e encontrada em lugares úmidos ou inundados, desenvolvendo-se preferencialmente em solo de boa fertilidade, areno-argiloso ou argiloso e com bom teor de matéria orgânica. É originária da Ásia e encontra-se aclimatada no Brasil em todos os Estados, particularmente no Rio Grande do Sul, Sul e Sudeste (Lopes et al., 2000). A planta inteira é utilizada na medicina popular, principalmente como antidisentérica, antisséptica, detersiva, estomáquica, diurética, antitérmica, anti-reumática, vermífuga e excelente cicatrizante de feridas e úlceras. Também é fortemente emenagoga e abortiva, não sendo recomendada para gestantes (Morgan, 1984). Suas propriedades terapêuticas são úteis também no combate do câncer (Fukuyama et al., 1983), assim como nos problemas circulatórios, pois estimula a circulação e diminui a fragilidade capilar, tendo efeito hemostático, bastante útil no tratamento de hemorróidas (Teske \& Trentini, 1995).

Apesar de sua enorme importância como planta medicinal, a espécie carece ainda de estudos relacionados ao seu comportamento genotípico e fenotípico. Assim, diante dessa falta de informações a respeito dos aspectos genéticos desta espécie, o presente trabalho teve como objetivo estabelecer padrões isoenzimáticos para caracterização de oito acessos, com a finalidade de investigar polimorfismos isoenzimáticos em

\footnotetext{
${ }^{1}$ Parte da tese de mestrado na área de genética e melhoramento de plantas. Depto. Fitotecnia, UFV, Viçosa-MG.
} 
P. punctatum Ell., bem como, obter estimativas da diversidade genética entre os acessos por meio desses padrões isoenzimáticos.

\section{MATERIAL E MÉTODOS}

Na caracterização de oito acessos de P. punctatum Ell. foram utilizadas mudas jovens obtidas pelo método de mergulhia, oriundas de São Paulo (SP), Peruíbe (SP), Viçosa (MG), Raul Soares (MG) e Canaã (MG). Os acessos foram assim identificados: VÇA e VÇB (Viçosa-MG), SPA (São Paulo-SP), SPB (Peruíbe-SP), RSA, RSB e RSC (Raul Soares-MG) e CAN (Canaã-MG).

As plantas obtidas de mudas dos acessos originais foram cultivadas em vasos de polietileno de $5 \mathrm{~L}$, preenchidos com substrato solo/esterco/areia, na proporção de 3:2:1, e mantidas em casa de vegetação.

As análises isoenzimáticas foram realizadas em laboratório na UFV. Utilizou-se a técnica de eletroforese horizontal em gel de amido a $12 \%$, para determinar os fenótipos isoenzimáticos da malato desidrogenase (MDH), esterase (EST), glutamato desidrogenase (GDH), glutamato oxaloacetato transaminase (GOT), fosfatase ácida (ACP), peroxidase (PO), leucina aminopeptidase (LAP), álcool desidrogenase (ADH), isocitrato desidrogenase (IDH) e chiquimato desidrogenase (SKDH). As amostras, constituídas por folhas jovens situadas na porção apical, foram maceradas em almofariz de porcelana previamente resfriado e mantido à baixa temperatura. O macerado foi absorvido em retângulos de papel cromatográfico Whatman $3 \mathrm{M}$ e aplicados nos géis. Realizou-se uma pré-corrida, a $4^{\circ} \mathrm{C}$, com corrente constante a $150 \mathrm{~V}$, por 30 minutos, após a qual, retiraram-se os retângulos de papel e ajustou-se a voltagem para 300 $\mathrm{V}$, exceto para o sistema peroxidase, em que se utilizou $200 \mathrm{~V}$, permanecendo assim até o final da corrida. A eletroforese foi conduzida em géis de amido de milho (Maizena) e amido hidrolisado (SIGMA). No preparo dos géis, empregaram-se $42 \mathrm{~g}$ de amido em $350 \mathrm{ml}$ de solução-tampão, de acordo com o proposto por Conkle et al. (1982).
A revelação da maioria dos sistemas foi realizada no escuro a aproximadamente $37^{\circ} \mathrm{C}$, com exceção da peroxidase, que foi efetuada à temperatura de $8^{\circ} \mathrm{C}$, aproximadamente. Após a revelação, os géis foram secados pelo método "bastidor", de acordo com o procedimento descrito por Alfenas et al. (1991). A descrição genética das bandas procedeu-se conforme metodologia proposta por Neale et al. (1984).

Para estimar a distância genética entre os acessos a partir de dados isoenzimáticos, utilizou-se a metodologia da análise multivariada do índice de similaridade de Jaccard e o agrupamento de Tocher, calculados pelo programa GENES. O índice de Jaccard é utilizado para fenótipos não interpretáveis em níveis de locos e alelos, por ser próprio para dados do tipo presença/ausência de bandas isoenzimáticas (Alfenas, 1991).

Segundo Sneath \& Sokal (1973), o índice de similaridade de Jaccard pode ser definido pela fórmula:

$$
\text { Iii, }=\frac{a}{a+b+c}
$$

em que a = número de casos em que a banda está presente nos dois acessos, simultaneamente; $b=$ número de casos em que a banda está presente somente no acesso $\mathrm{i}$; $\mathrm{c}=$ número de casos em que a banda está presente somente no acesso i,.

Foram utilizadas somente bandas cuja revelação era de interpretação consistente, descartando-se as bandas atribuídas a artefatos.

O método proposto por Tocher, citado por RAO (1952), requer a obtenção da matriz de dissimilaridade, em que a dissimilaridade foi obtida pelo complemento aritmético do índice de similaridade de Jaccard, utilizando-se a seguinte expressão:

$$
\text { CIii, = } 1 \text { - Iii, }
$$

em queCIii, = medida de dissimilaridade; e Iii, = índice de Jaccard.

Por meio da matriz de dissimilaridade, foi identificado o par de acessos mais similar, formando-se o grupo inicial. Para inclusão de novos acessos no grupo, compararam-se o acréscimo no valor médio da distância dentro do grupo e o nível máximo permitido (a) para que a distância média intragrupo fosse inferior a qualquer distância intergrupo.

\section{RESULTADOS E DISCUSSÃO}

\section{Caracterização Isoenzimática dos Acessos}

O amido de milho mostrou-se eficiente na separação das bandas isoenzimáticas da IDH, ADH e $\mathrm{MDH}$, permitindo a revelação de bandas nítidas e com fácil identificação. Esse meiosuporte foi utilizado com grande eficiência por Amaral Júnior (1994). Entretanto, para os demais sistemas, o amido hidrolisado apresentou resolução superior à do amido de milho. Esse fato pode ter ocorrido em razão do melhor controle de qualidade para produção de amido hidrolisado (Shuelter, 1996).

Todos os sistemas enzimáticos estudados, com exceção do sistema LAP, apresentaram elevado polimorfismo com ausência e presença de bandas (Figura 1).

Com relação à $\mathrm{MDH}$, foram detectadas nove bandas polimórficas, reunidas praticamente na mesma região de atividade, resultando em quatro padrões isoenzimáticos. Os acessos VÇA, CAN, RSA, RSB e SPB apresentaram cinco bandas isoenzimáticas correspondente ao padrão 1, enquanto que os acessos SPA (padrão 2), RSC (padrão 3) e VÇB (padrão 4), mostraram padrão individual de bandeamento com cinco e quatro bandas, respectivamente. Pode-se evidenciar um grau de parentesco muito próximo dos acessos do padrão 1 , uma vez que apresentavam semelhanças.

O sistema ACP apresentou atividade anódica com a presença de 11 bandas, que, basicamente, se distribuem em duas regiões de atividade, as quais formam cinco padrões isoenzimáticos. $\mathrm{Na}$ região 1, de maior poder de migração, é possível deduzir a existência de um loco com dois alelos. Na região 2, supõe-se que haja vários alelos, onde se encontram bandas de difícil interpretação genética. $\mathrm{Na}$ discriminação dos acessos, segundo os padrões e as respectivas bandas eletroforéticas, observou a presença das bandas $7 ; 8 ; 9 ; 10$ e 11 somente nos acessos RSC (padrão 4) e VÇB (pa- 


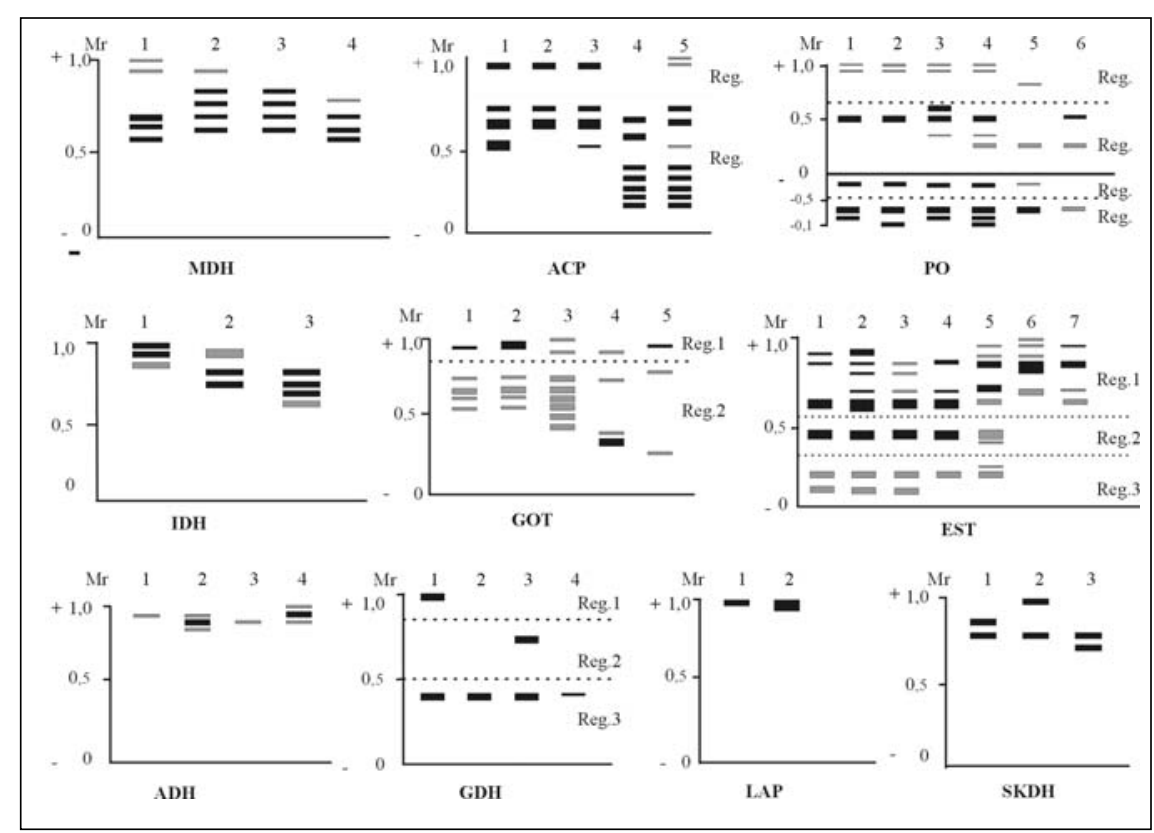

Figura 1. Padrões isoenzimáticos da enzima MDH, ACP, PO, IDH, GOT, EST, ADH, GDH, LAP e SKDH, obtidos do tecido foliar de oito acessos de Polygonum punctatum Ell. e sua mobilidade relativa (Mr). Viçosa, UFV, 1997.

drão 5), podendo significar a fixação dos alelos nesse loco. O acesso VÇB apresentou padrão individual de bandeamento com 10 bandas evidenciadas.

O sistema PO apresentou atividades anódica e catódica, enquanto os demais somente mostraram atividade anódica. Foram detectadas 11 bandas, sendo sete com migração anodal e quatro com migração catodal, distribuídas em quatro regiões de atividade. Neste sistema, o grande polimorfismo permitiu a separação de seis padrões isoenzimáticos. No padrão 1 foram agrupados três acessos (VÇA, RSA e SPB), enquanto os demais padrões apresentaram bandeamento individual para cada acesso. Os resultados obtidos confirmaram os estudos feitos em outras espécies (McDaniel, 1970; Smith et al., 1985), em que a peroxidase foi efetiva em caracterizar a maioria dos acessos. Em relação ao sistema IDH, foram constatados três padrões isoenzimáticos, evidenciando sete bandas, as quais apresentaram diferenças na intensidade de coloração e espessura. Os acessos VÇA, CAN, RSA, RSB, SPB e SPA correspondem ao padrão 1 , e os acessos RSC e VÇB ao padrão 2 e 3, respectivamente. Enquanto que, no sistema GOT foi observada a ocorrência de duas regiões de atividade, totalizando 13 bandas e cinco padrões isoenzimáticos. Na região 1 é provável a existência de apenas um loco com três alelos, enquanto que a região 2 mostrouse altamente polimórfica. Os padrões 1 (VÇA, RSA e RSB) e 2 (CAN e SPB) apresentaram o mesmo bandeamento com cinco bandas, diferindo apenas na espessura da banda 2. Os acessos SPA (padrão 3), RSC (padrão 4) e VÇB (padrão 5) apresentaram padrão individual de bandeamento com oito, quatro e três bandas, respectivamente.

O sistema EST apresentou elevado polimorfismo, sendo observada a presença de 13 bandas, distribuídas em três regiões de atividades. A região 1 , de maior migração, apresentou bandas em alguns padrões, mas ausentes em outros, bandas de mobilidade relativa muito próximas e bandas com diferenças na espessura e intensidade. A região 2, intermediária, e a região 3 , de baixa migração, parecem conter um loco com dois e três alelos, respectivamente. $\mathrm{O}$ grande polimorfismo permitiu a separação mais precisamente dos acessos em sete padrões isoenzimáticos. Esse polimorfismo encontrado no sistema esterase também foi observado em outras plantas, como Hordeum (McDaniel, 1970) e alfafa (Quiros, 1980).

A enzima $\mathrm{ADH}$ apresentou quatro bandas distribuídas em apenas uma re- gião de atividade, as quais resultaram em quatro padrões isoenzimáticos. Foi constatada a presença de três bandas nos padrões 2 (VÇB) e 4 (VÇB), sendo a intermediária mais espessa e com coloração mais intensa, o que permite supor ser o heterozigoto de uma enzima dimérica. Segundo Pasteur et al. (1988), as desidrogenases são enzimas diméricas, que podem apresentar mobilidade eletroforética muito próxima, com moléculas nos extremos e a forma heterozigota representada pelas bandas intermediárias, que freqüente-mente são mais intensamente coloridas do que as outras bandas. Assim, pode-se admitir que os padrões 1 (VÇA, CAN, RSA, RSB e SPB) e 3 (RSC) representaram o homozigoto dessa enzima dimérica. Com base no zimograma dos padrões isoenzimáticos para $\mathrm{GDH}$, podem-se hipotetizar três regiões de atividade, em virtude da distância de migração entre as bandas. $\mathrm{O}$ sistema apresentou baixo polimorfismo, evidenciando quatro padrões isoenzimáticos, no padrão 1 (VÇA, CAN, RSA, RSB e SPB) apresentou a banda 1 de maior migração, agrupando a maioria dos acessos. Os acessos SPA (padrão2), RSC (padrão 3) e VÇB (padrão 4) apresentaram padrão individual de bandeamento. O sistema LAP apresentou apenas uma região de atividade, com bandas de espessuras diferentes. Em razão do pouco polimorfismo, o método foi ineficiente para discriminar os acessos, possibilitando apenas a distinção de dois padrões.

No sistema SKDH foi detectada a presença de três padrões isoenzimáticos, apresentando quatro bandas reunidas praticamente em apenas uma região de atividade. A maioria dos acessos foi agrupado no padrão 1 (VÇA, CAN, RSA, RSB, SPB e RSC) e os acessos SPA (padrão 2) e VÇB (padrão 3) também apresentaram padrão individual de bandeamento.

De modo geral, foi amplo o polimorfismo, permitindo estabelecer padrões individuais para todos acessos. Pode-se supor que a utilização de vários sistemas enzimáticos contribui para evidenciar tal polimorfismo, demonstrando o potencial das isoenzimas como marcadores genéticos no gênero Polygonum. 
Quadro 1. Distribuição de oito acessos de Polygonum punctatum Ell. nos padrões de bandeamento de ACP, IDH, PO, MDH, SKDH, EST, ADH, GOT e GDH. Viçosa, UFV, 1997.

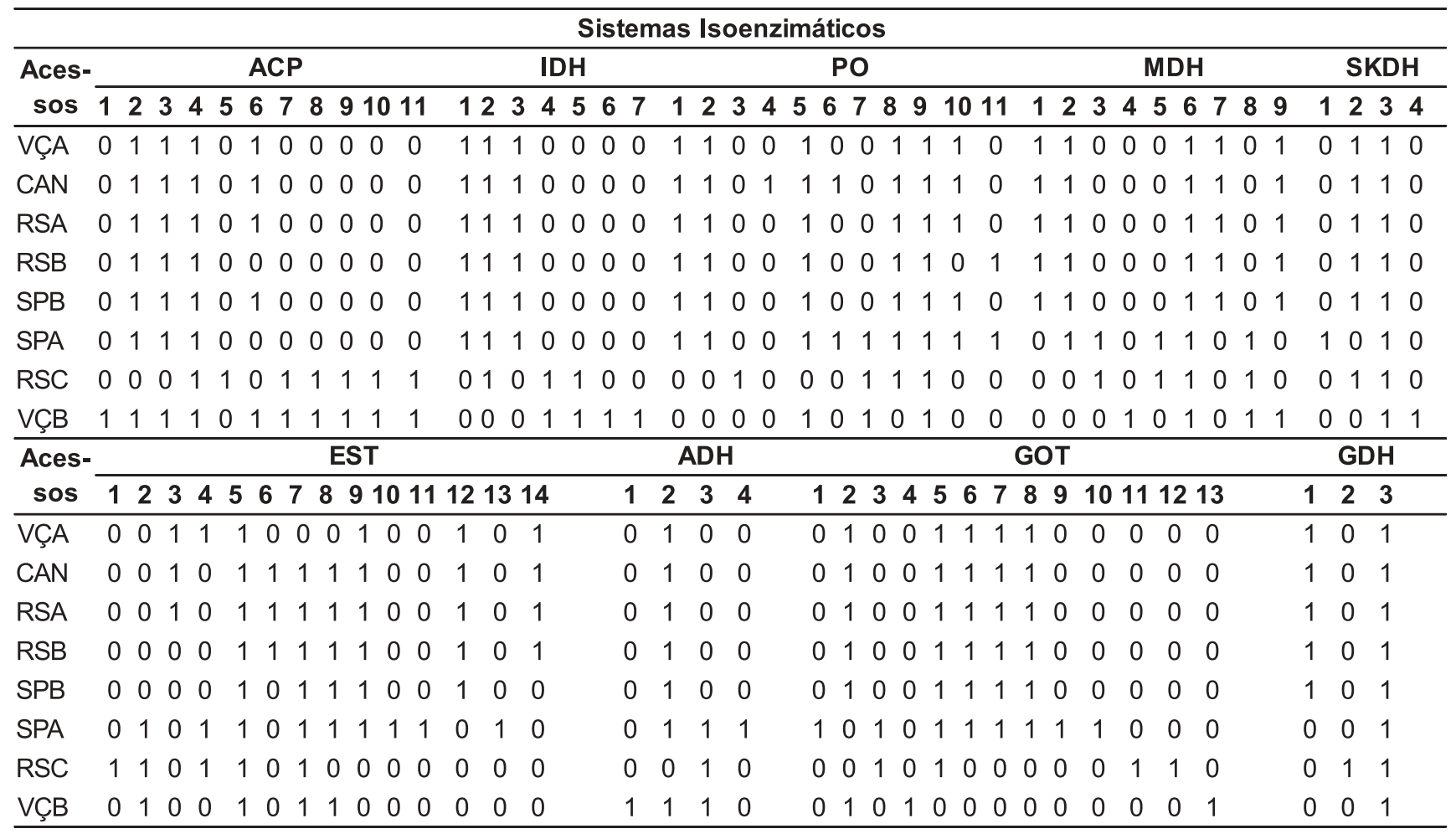

Quadro 2. Medidas de dissimilaridade obtidas a partir de complemento aritmético do índice de Jaccard entre os padrões de bandas isoenzimáticas de oito acessos de Polygonum punctatum Ell. Viçosa, UFV, 1997.

\begin{tabular}{lcccccccc}
\hline Acessos & VÇA & CAN & RSA & RSB & SPB & SPA & RSC & VÇB \\
\hline VÇA & - & 0,1538 & 0,1081 & 0,2105 & 0,1389 & 0,5472 & 0,8000 & 0,7636 \\
CAN & & - & 0,0526 & 0,1538 & 0,1316 & 0,5273 & 0,8136 & 0,7368 \\
RSA & & & - & 0,1081 & 0,0833 & 0,5370 & 0,8070 & 0,7273 \\
RSB & & & & - & 0,1389 & 0,5192 & 0,8000 & 0,7407 \\
SPB & & & & & - & 0,5098 & 0,7963 & 0,7115 \\
SPA & & & & & & - & 0,6842 & 0,7377 \\
RSC & & & & & & & - & 0,6250 \\
VÇB & & & & & & & & - \\
\hline
\end{tabular}

VÇA - Viçosa (MG), CAN - Canaã (MG), RSA - Raul Soares (MG), RSB - Raul Soares (MG), SPB - Peruíbe (SP), SPA - São Paulo (SP), RSC - Raul Soares (MG) e VÇB - Viçosa (MG).

Na maioria dos sistemas enzimáticos, houve grande variação no número, na intensidade e na espessura das bandas. Tal variação pode estar relacionada com o grau de ploidia na espécie. Segundo Gottlieb (1982), a diferença no grau de ploidia pode ser observada no gel, pela presença de um número maior de bandas. Por sua vez, Messina et al. (1991) afirmaram que essa diferença pode ser averiguada pelo aumento da intensidade e da espessura das bandas causado pela variação na dose de alelos presentes.
Análise da Diversidade Genética dos Acessos por Padrões Isoenzimáticos

Alguns sistemas enzimáticos apresentaram grande número de bandas. Assim, para visualizar melhor os sistemas, foi elaborado quadro de distribuição dos acessos e as respectivas bandas, adotando-se os números 0 e 1 para indicar ausência e presença de bandas, respectivamente (Quadro 1).

Os sistemas isoenzimáticos ACP, IDH, PO, MDH, SKDH, EST, ADH, GOT e GDH apresentaram elevado polimorfismo com presença e ausência de bandas. A enzima LAP não foi considerada, por ter apresentado baixo polimorfismo nos acessos estudados. Foi verificado que os acessos VÇA, CAN, RSA, RSB e SPB apresentaram o mesmo padrão de bandeamento para os sistemas IDH, MDH, SKDH, ADH, GOT e GDH. Entretanto, nos demais sistemas houve grande variação nos padrões (Quadro 1).

Em razão do fato de os acessos não apresentarem padrões isoenzimáticos 
Quadro 3. Limite de distância intergrupo, com base nas medidas de dissimilaridade entre pares de acessos de Polygonum punctatum Ell. Viçosa, UFV, 1997.

\begin{tabular}{cc}
\hline Acessos & Distância mínima \\
\hline VÇA & 0,000 \\
CAN & 0,000 \\
RSA & 0,000 \\
RSB & 0,000 \\
SPB & 0,000 \\
SPA & 0,000 \\
RSC & 0,154 \\
VÇB & 0,108 \\
Maior distância entre as Mínimas (a) & 0,154 \\
\hline
\end{tabular}

Quadro 4. Agrupamento pelo método de otimização de Tocher a partir dos dados isoenzimáticos de acessos de Polygonum punctatum Ell. Viçosa, UFV, 1997.

\begin{tabular}{cc}
\hline GRUPOS & ACESSOS \\
\hline$I$ & VÇA, CAN, RSA, RSB, SPB, SPA \\
II & VÇB \\
III & RSC \\
\hline
\end{tabular}

idênticos, procedeu-se ao estudo da similaridade genética, envolvendo a combinação dos oito acessos, tomados dois a dois. Por meio do índice de similaridade de Jaccard, obteve-se a estimativa da dissimilaridade entre os acessos a partir de dados isoenzimáticos (Quadro 2). Os valores das medidas de dissimilaridade variaram amplamente entre 0 e 0,8136 . Os acessos VÇA, CAN, RSA, RSB, SPB e SPA foram os mais similares e o par que apresentou maior dissimilaridade foi constituído pelos acessos RSC e VÇB. Ambos diferiram amplamente dos demais acessos (Quadro 2).

Na aplicação do método de agrupamento por otimização, considerando-se a maior distância no conjunto das menores distâncias (a) entre cada acesso, o valor obtido foi 0,154 (Quadro 3). Dessa forma, houve formação de três grupos (Quadro 4): o grupo I contendo seis acessos (VÇA, CAN, RSA, RSB, SPB e SPA) e os grupos II e III, os acessos VÇB e RSC, respectivamente. Desta forma, foi evidenciado o baixo grau de diversidade genética dando suporte à hipótese de origem comum dos acessos, com grau muito próximo de parentesco (Lopes, 1997). Entretanto, a variabilidade entre os padrões pode ter decorrido da forte pressão de seleção imposta sobre esses sistemas, com objetivo de favorecer determinados produtos gênicos mais eficiente. Tal fato justifica a grande facilidade da espécie Polygonum punctatum Ell. de adaptarse à diversas condições ambientais. Segundo Sultan \& Bazzaz (1993), outras espécies do gênero Polygonum apresentam elevado grau de plasticidade fenotípica, sendo este o principal meio de adaptação individual dessas plantas ao ambiente.

\section{LITERATURA CITADA}

ALFENAS, A.C.; PETERS, I.; BRUNE, W.; PASSADOR, G.C. Eletroforese de proteinas e isoenzimas de fungos e essências florestais. Viçosa, UFV, 1991. 242 p.

AMARAL JÚNIOR., A.T.; CASALI, V.W.D.; ALFENAS, A.C. BRUNE, W. Análise isozimática de acesso de moranga. Horticultura Brasileira, Brasília, v. 12, n. 1, p. 37-39,1994.

CONKLE, M.T.; HODGSKISS, P.D.; NUNNALLY, L.B.; IMRIE, B.C. Starch gel electrophoresis of conifer seeds: a laboratory manual. Berkeley: USDA, Forest Service, 1982. 18 p. (Gen. tech. rep., PSW-64).

CRUZ, C.D. Aplicação de algumas técnicas multivariadas no melhoramento de plantas. Piracicaba: ESALQ, USP, 1990. 188 p. ESALQ, 1990. biométricos aplicados ao melhoramento genético. Viçosa, MG: UFV, 1994. 390 p.
CRUZ, C.D.; REGAZZI, A.J. Modelos
FIGUEIREDO-RIBEIRO, R.C.L.; DUDLEY, J.W. Morphology and essential oil in the taxonomy of Ocimum nudicaule Benth. (Labiatea). Anais de Academia Brasileira de Ciências, v. 32, p. 65-71, 1986. FUKUYAMA, Y.; SATO, T.; MIURA, I.; ASAKAWA, Y. Hidropiperoside, a novel coumaryl glycoside from the root of Polygonum hidropiper. Phytochemistry, v. 22, p. 549-552, 1983.

GOTTLIEB, L.D. Conservation and duplication of isozyme in plants. Science, v. 216, p. 373-379, 1982.

LOPES, R.C. Caracterização isozimática, diversidade genética e produção de óleo essencial em acessos de Polygonum punctatum Ell. Viçosa, MG: UFV, 1997. 88 p. (Tese mestrado), UFV, 1997. LOPES, R.C.; ANDRADE, F.M.C.; CASALI, V.W.D. Plantas medicinais e aromáticas: ervade-bicho e chambá. Viçosa, UFV, 2000. $149 \mathrm{p}$.

MARDIA, K.V., KENT, J.T., BIBBY, J.M. Multivariate analysis. London: Academic Press, $1979.512 \mathrm{p}$.

McDANIEL, R.G. Electrophoretic characterization of proteins in Hordeum. Journal of Heredity, v. 61, p. 243-247, 1970.

MESSINA, R.; TESTOLIN, R.; MORGANTE, M. Isozymes for cultivar identification in Kiwifruit. HortScience, v. 26, p. 899-902, 1991.

MORGAN, R. Plantas medicinais. São Paulo: Hemus, 1984. 555 p.

NEALE, D.B.; WEBER, J.C.; ADAMS, W.T. Inheritance of needle tissue isozymes in douglasfir. Canadian Journal of Genetics and Cytology, v. 26, p. 459-468, 1984.

PASTEUR, N.; PASTEUR, G.; BONHOME, F. Pratical isozyme genetics. New York: Halsted Press, 1988. 255 p.

QUIROS, C.F. Identification of alfafa plants by electrophoresis. Crop Science, v. 20, p. 262-264, 1980. RAO, A.V.; PRASAD, A.S.R.; SAIKRISHNA, T. Genetic divergence among some brown plant hopper resistant rice varieties. The Indian Journal of Genetics \& Plant Breeding, v. 4, p. 179-185, 1981.

RAO, R.C. Advanced statistical methods in biometric research. New York: John Wiley and Sons, $1952.390 \mathrm{p}$.

SHUELTER, A.R. Análise isozimática, dialélica e diversidade genética da Pimenta Silvestre (Capsicum flexuosum Sendt.). Viçosa, MG: UFV,1996. $80 \mathrm{p}$.

SNEATH, P.H.; SOKAL, R.R. Numerical taxonomy the principles and practice of numerical classification. San Francisco: W.H. Freeman, $1973.513 \mathrm{p}$.

SMITH, J.C.S.; GOODMAN, M.M.; STUBER, C.N. Genetic variability within maize germoplasm. II. Widely used inbred lines 1970 to 1979. Crop Science, v. 25, p. 681-685, 1985.

SUGIYARTO, E.; SOEMARTONO, D.; MANGOENDIDJOJO, W. Yield stability analysis in sugarcane cultivar trial Agricultural Science, v. 3, p. 315-322, 1984.

SULTAN, S.E.; BAZZAZ, F.A. Phenotypic plasticity in Polygonum persicaria. I. Diversity and uniformity in genotypic norms of reaction to light. Evolution. v. 47, p. 1.009-1.031, 1993.

TANKSLEY, S.D.; ORTON, T.J. Isozimes in plant genetics and breeding. Amsterdam: Elseveir, 1983. $516 \mathrm{p}$.

TESKE, M.; TRENTINI, A.M.M. Compêndio de fitoterapia. Curitiba: Herbarium Laboratório Botânico, 1995.317 p.

TORGGLER, M.G.F. Isoenzimas: variabilidade genética em plantas. Sociedade Brasileira de Genética, Ribeirão Preto, 1995. 186 p. 